\title{
Atomic Force Microscopy Imaging of Living Cells
}

\author{
Alexandre Berquand, ${ }^{1}$ * Charles Roduit, ${ }^{2}$ Sandor Kasas, ${ }^{2}$ Andreas Holloschi, ${ }^{3}$ \\ Leslie Ponce, ${ }^{3}$ and Mathias Hafner ${ }^{3}, 4$ \\ ${ }^{1}$ Veeco Instruments GmbH, Dynamostrasse 19, 68165 Mannheim, Germany \\ ${ }^{2}$ Institut de Physique des Systèmes Biologiques, Ecole Polytechnique Fédérale de Lausanne (EPFL), Lausanne, Switzerland \\ ${ }^{3}$ University of Applied Sciences, Institute of Molecular and Cell Biology, Mannheim, Germany \\ ${ }^{4}$ Medical Faculty Mannheim, University of Heidelberg, Mannheim, Germany \\ *aberquand@veeco.de
}

\section{Introduction}

Over the last two decades, Atomic Force Microscopy (AFM) has emerged as the tool of choice to image living organisms in a near-physiological environment. Whereas fluorescence microscopy techniques allow labeling and tracking of components inside cells and the observation of dynamic processes, AFM is mainly a surface technique that can be operated on a wide range of substrates including biological samples. AFM enables extraction of topographical, mechanical and chemical information from these samples.

This review gives a brief overview of the existing AFM modes and the accessories that can be used to extract various types of data from living cells. Although AFM can provide important information from cells, it now appears essential to couple it with light microscopy techniques like bright-field, differential interference contrast (DIC) [1], epifluorescence [2-4], or total internal reflection fluorescence (TIRF) [5]. The present review will also highlight how such a combination is possible.

\section{Instrumentation}

Setup. For a non-compromised coupling with light optics, the ideal AFM must fulfill some technical requirements (Figure 1A). First, the head must allow large and easy access to the sample (to exchange the media) and be compatible with most types of condensors. Second, the sample insert should be physically compatible with the major types of supports used both in AFM and light microscopy techniques (glass slides, cover slips, and Petri dishes having different diameters). Third, it should contain a hole in its center to ensure propagation of the light beam, which should be as large as possible to allow the user freedom to operate with high-magnification objectives. The stage should also have temperature control capabilities to keep the sample at a controlled temperature. Of course, coupling with light optics should not compromise any AFM operation. (AFM modes will be detailed further on.)

In regular incubators, cells are maintained under a $\mathrm{CO}_{2} / \mathrm{O}_{2}$ gas flow, and culture media are buffered by bicarbonate so they can be kept alive over a non-limited period of time. However, out of the incubator, the culture medium must be replaced to keep the $\mathrm{pH}$ constant. Some advanced perfusion systems enable long-duration culture of cells while maintaining full compatibility with the AFM (Figure 1B). By using one those specific chambers, cell culture media can be perfused continuously or intermittently, either to replace nutrient-depleted media or to investigate a dynamic response to a change in medium composition. The system must also be totally sealed to avoid evaporation and allow for perfusion of the gas. Eventually, the temperature must be precisely controlled, especially close to the area where the sample is being scanned. Together, these features can maintain cell cultures effectively for many hours or even days under the appropriate conditions.

Cells can be adherent or not adherent to the substrate used for imaging. For adherent cells, the cells are grown directly on the imaging support. If certain cells are found to be biocompatible on a wide range of surfaces, it is strongly advised to use a transparent substrate like glass, which can combine AFM and light optical measurements. In the case of non-adherent cells,
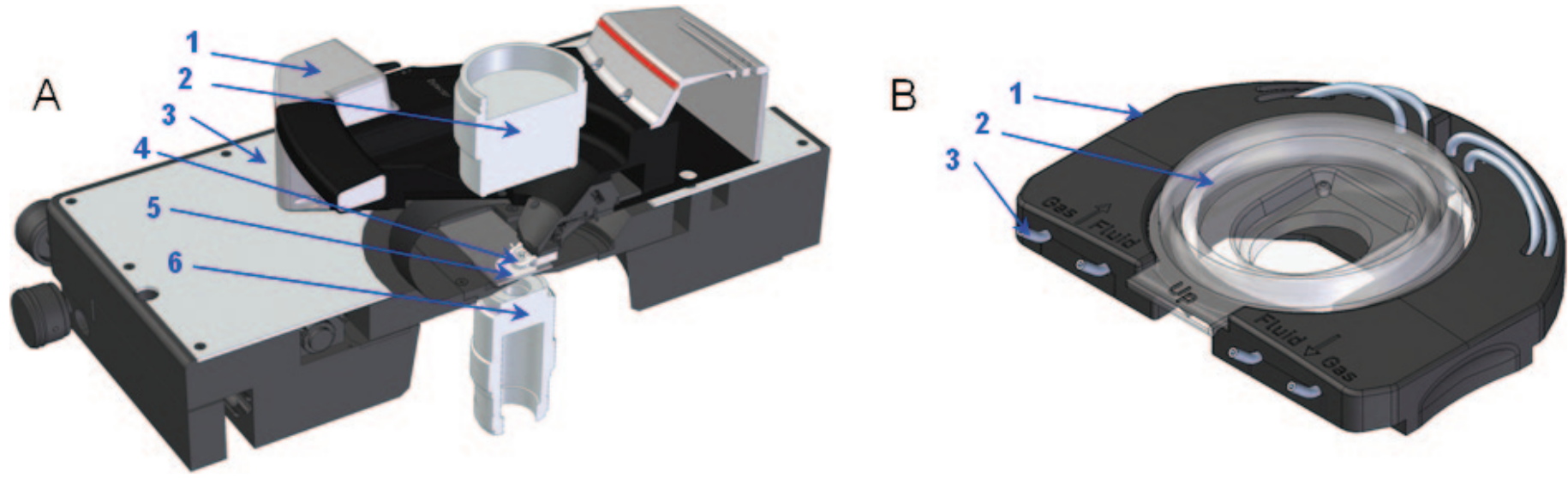

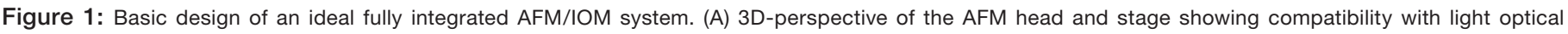

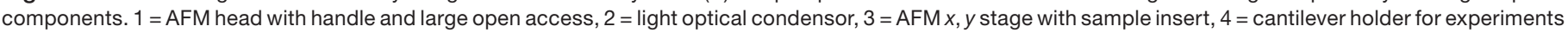

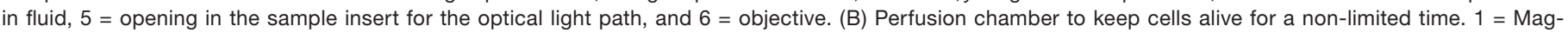
netic clamp that attaches to the AFM stage, $2=$ silicon lid that seals the chamber and fits with the cantilever holder, and $3=$ inlets and outlets for liquid and gas. 

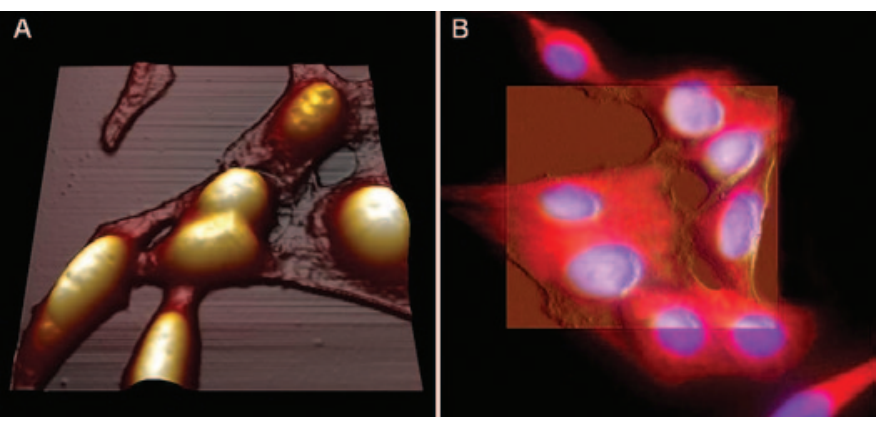

Figure 2: Typical imaging performance of a fully integrated AFM/IOM system. (A) Typical $100 \times 100 \times 5 \mu \mathrm{m} 3 \mathrm{~d}$-height contact mode image of living U2-OS cells achieved at $0.6 \mathrm{~Hz}$ and medium resolution $(256 \times 256)$. Using ScanAsyst software, images can be captured in about 7 minutes. (B) Typical overlay of AFM (contact mode, deflection channel, $75 \times 75 \times 3 \mu \mathrm{m}$ ) and fluorescence (actin microfilaments were stained with Phalloidin-Alexa488 [red] and cell nuclei with DAPI [blue]) images of Hela cells providing both AFM and optical information simultaneously. Image width $=120 \mu \mathrm{m}$.

several techniques can be used. A more elegant one consists of trapping small eukaryotic cells or bacteria in pores by filtration [6]. Having an AFM directly correlated to an inverted light microscope allows a straightforward localization. This technique has been proven to give valuable results on living red blood cells, yeasts, and bacteria.

Operating modes. The most commonly used AFM modes are the contact [7] and tapping [8] modes, though the force volume mode can also be considered as an imaging technique because topographical information can be extracted from force volume images. In contact mode the AFM probe is scanned over the sample surface at a constant force, and if the scanning forces are not carefully adjusted, shear and friction effects can cause delicate samples to be damaged. In the tapping mode, the probe oscillates in the vertical axis and only intermittently contacts the sample, thus friction forces are negligible, which makes it one of the techniques of choice to image living cells. Whichever technique is used, working on biological samples requires choosing the proper cantilevers.
Silicon nitride cantilevers give very good results on most biological samples [9-10]. The spring constant should be as low as possible in contact mode, whereas stiffer levers can be used in tapping mode. Adjusting the imaging forces requires calculating the deflection sensitivity and the spring constant (see "Force mode" paragraph). In tapping mode, the cantilever must oscillate close to its resonance frequency. The Auto Tune function of the microscope can help find the proper working frequency [11]. As cells are extremely soft substrates, the best lateral resolution that can be expected is about $10 \mathrm{~nm}$ [12-14]. Both of these modes have been shown to provide good results in live-cell imaging (Figure 2A), but more relevant results are obtained when combined with light microscopy (Figure 2B).

In force mode, the AFM tip is first located above the sample, lowered to come in contact with it, and then retracted. During this approach-retract cycle, the probe goes through an attractive field, reaches the contact point, and indents into the sample. The effects can be measured and plotted. The linear portion of this extend curve can be extrapolated to estimate the sample stiffness and calculate the corresponding Young's modulus using a mathematical fit. When the tip moves back to its original position, the rupture of the adhesive meniscus existing between the tip and the sample causes a jump in the retraction curve. This step height represents the non-specific adhesion between the probe and the sample surface and is greatly reduced when working in a fluid environment. The deflection sensitivity and the spring constant of the cantilever, required to access quantitative values, can be automatically calculated with most modern AFMs. The force mode can be operated either by using single force measurements or repeated on a matrix of points and thus will be referred to as the force volume mode. At each pixel of the image, both the extend curve and the retract curve are recorded, allowing simultaneous adhesion and stiffness mapping (Figure 3).

AFM probes can also be functionalized with a wide range of active ligands to investigate the specificity of interaction toward the corresponding receptors, most of the time distributed on

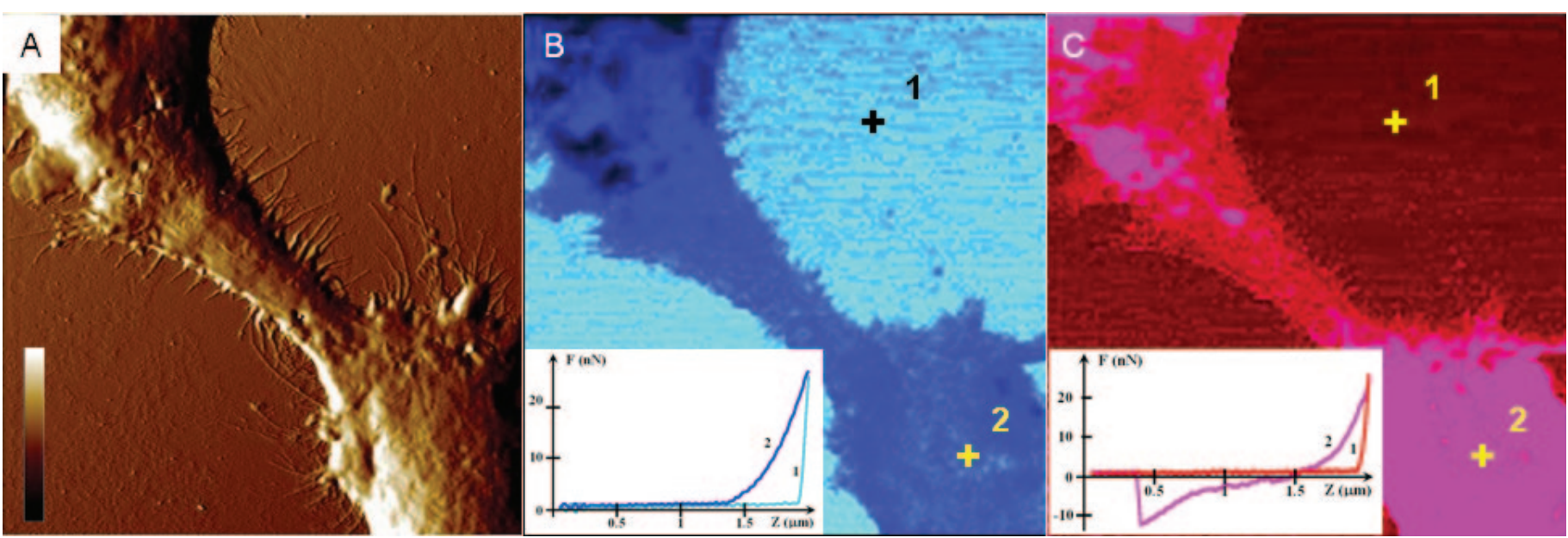

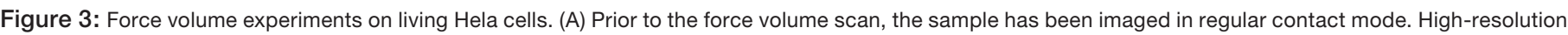

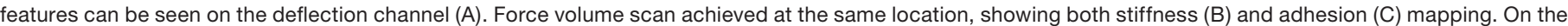

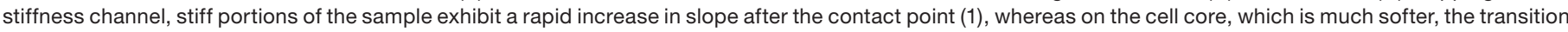

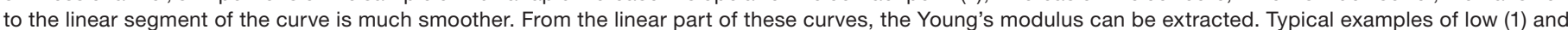

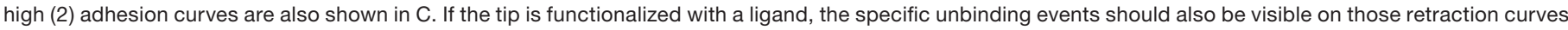
and can be quantified by using specific programs (see Figure 4). 
the topmost layer of the cells. In this case, specific unbinding events can be visualized on the retraction curve and distinguished from non-specific adhesion. This technique can also be used to map the distribution of molecules of interest inside a plasma membrane or even determine the kinetic affinity constant between two single molecules $[15,16]$. Force volume images can be post-processed, and different types of data can be extracted from them. Certain programs allow display of a three-dimensional rendering of the sample topography, where a color scale may be added as a second skin to display the elasticity. If the tip is functionalized, red arrows can indicate the location of the specific unbinding events (Figure 4). As in contact or tapping modes, force measurements can also be combined with light optical imaging. Overlaying light and AFM images provides correlation of specific parts of the cell with local changes in topography, chemistry, and mechanical properties [17].

Recently the Peak Force Tapping ${ }^{\mathrm{TM}}$ mode has been developed, in which the system modulates the $z$-piezo far below the cantilever resonance frequency and performs a very fast curve at each pixel of the image. The peak interaction force of each of these curves is used as the imaging feedback signal, enabling operation at lower forces than even what is achievable with normal tapping mode, and the different channel signals (like topography, peak force error, adhesion, elastic modulus, deformation, and dissipation) may be calculated. Another key feature of this mode is that quantitative information can be directly extracted from all the images if the probe is calibrated on a known sample prior to the experiment. This derivative mode, referred to as Peak Force QNM (Quantitative Nanomechanical properties), is faster than any force mode and provides more information than tapping mode and force volume mode.

Living cells. Since the first observations of dynamical behavior [18] and the first qualitative [19] and quantitative [20] measurements of local mechanical properties, SPM techniques have greatly improved, and AFM has shown itself to be a straightforward technique to investigate living cells. Whereas electron microscopy and fluorescence microscopy techniques require sample modification, AFM can be operated on the raw sample in nearly physiological conditions. Moreover, whereas electron microscopy implies working on a "dead" sample, AFM can be performed on living cells and can be combined with fluorescence microscopy with a remarkable precision. Combining the AFM approach, for locally applied mechanical deformations, with live cell fluorescence imaging allows the simultaneous measurement of biochemical signaling responses [21].

Topography. The primary function of AFM, however, is to obtain topographical information of the scanned sample. For instance, although the in-vitro structure of S-layers (monomolecular layers of proteins and glycoproteins found in the cell envelope of some bacteria) has been well established over the last decade, their direct imaging in living bacteria had never been adequate. Recently an in-vivo AFM investigation of S-layer nanoarrays [22] provided high-resolution images that open the way to important experiments in nanoscience. Without a doubt, the most unique aspect of AFM is its ability to establish the connection between the structure and the function of subcellular components, cells, and even tissues. For instance, a study of the ultrastructure of bone marrow CD34(+) cells pointed out that AFM might become a valuable tool for fast leukemic diagnosis and hematopoietic cell selection [23]. In endocrinology, AFM investigations of plasma membranes of living cells provided new insights into the mechanisms of docking and fusion of secretory vesicles and confirmed the existence of the porosome. This type of discovery can lead to amelioration of secretory defects in disease states [24]. In the field of regenerative medicine, using human embryonic stem (hES) cells, AFM was successfully used to show the specificity of distribution of TRA-1-81 receptors and revealed that differentiated and non-differentiated cells exhibit different distributions [25]. This technique can be extended to other cell types and offers exciting outlooks in several disciplines, tissue engineering among them.

Cell elasticity. Measurements of local changes in cell elasticity in response to various factors proved that softening or stiffening effects directly impact the structure and function of the cell and sometimes affect the organism at a higher scale. Early AFM studies from the beginning of the 1990s demonstrated that some dynamic rearrangements of the cytoskeleton enabled the cell to divide, migrate, or maintain shape, and that actin had a key role in these functions [26-33]. From a physiological point of view, it is known that an excess of plasma sodium can cause stroke, and recent AFM cell elasticity measurements achieved on living endothelial cells have shown a direct correlation between the sodium concentration
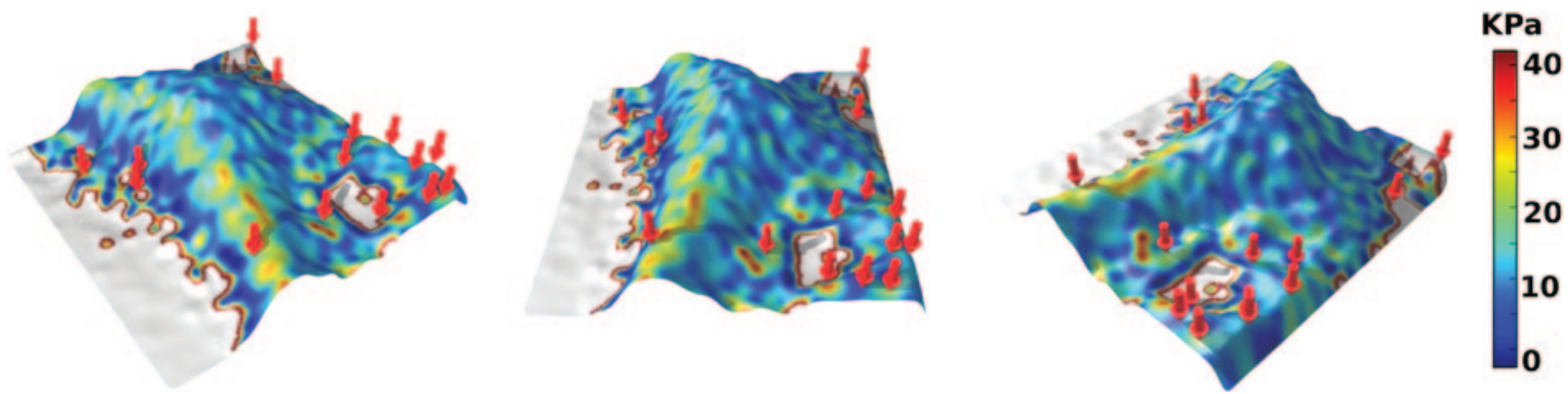

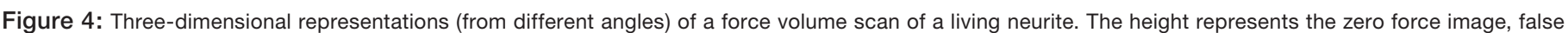

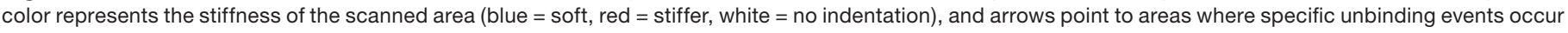
between aerolysin that is present on the tip and GPI-anchored proteins that are present on the sample. Scanned area $=5 \times 5 \mu \mathrm{m}$. 
and the cell stiffness [34]. Similar studies carried out on Hela and osteosarcoma cells showed the usefulness of combining fluorescence microscopy with AFM imaging and force measurements [17] for similar investigations. It is now well accepted that significant alterations of cell mechanical properties can lead to aberrant cellular processes and progression of diseases like cancer, malaria, and possibly neurodegeneration [35-41]. Thus the AFM, especially when operated in force mode, can be considered a powerful technique to assess and correlate the effects of aging, pathology, and drug treatment [30, 42-44]. Nevertheless, up to now, AFM force spectroscopy has suffered from two main drawbacks: the lack of spatial resolution and the fact that data acquisition and post-processing are highly time-consuming. Also, optimization of imaging parameters requires some expertise.

Ease of use. The AFM can now be used in fully automated mode, which makes imaging of living cells accessible to inexperienced users. ScanAsyst ${ }^{\mathrm{TM}}$ mode offers a good solution for both AFM beginners and advanced users. In that mode, algorithms continuously monitor the image quality to make appropriate parameter adjustments by using Peak Force Tapping mode technology.

\section{Materials and Methods}

All presented data were acquired on a Bioscope Catalyst ${ }^{\mathrm{TM}}$ (Veeco Instruments, Santa Barbara CA, USA). The light microscope employed was a DMI6000 (Leica, Wetzlar, Germany). Images in Figures 2, 5, and 6 were acquired using ScanAsyst ${ }^{\mathrm{TM}}$ and a Perfusing Stage Incubator ${ }^{\mathrm{TM}}$ (Veeco Instruments, Santa Barbara CA, USA). Images in Figures 5 and 6 were produced using Peak Force Tapping (Veeco Instruments, Santa Barbara CA, USA). For images captured in contact mode and force volume modes, MLCT cantilevers $(0.01 \mathrm{~N} / \mathrm{m})$ were used. SCANASYSTFLUID cantilevers $(0.4 \mathrm{~N} / \mathrm{m})$ (Veeco Instruments, Santa Barbara CA, USA) were used for peak force tapping images. Tips were functionalized as previously described [45].

Cultured and labeled U2-OS-osteosarcoma cells were prepared as previously described [17]. Neurons were prepared and cultured as previously described [46]. Cells were plated in 35-mm Petri dishes at a density of 50,000/dish. For AFM measurements, cells were maintained in a $\mathrm{K} 5$ medium (128 $\mathrm{mM} \mathrm{NaCl}, 5 \mathrm{mM} \mathrm{KCl}, 2.7 \mathrm{mM} \mathrm{CaCl}_{2}, 1 \mathrm{mM} \mathrm{MgCl}_{2}, 10 \mathrm{mM}$ glucose, $20 \mathrm{mM}$ HEPES [pH 7.4]) at room temperature.

Force volume data were post-processed using OpenFovea (www.freesbi.ch/openfovea). Each force distance curve was
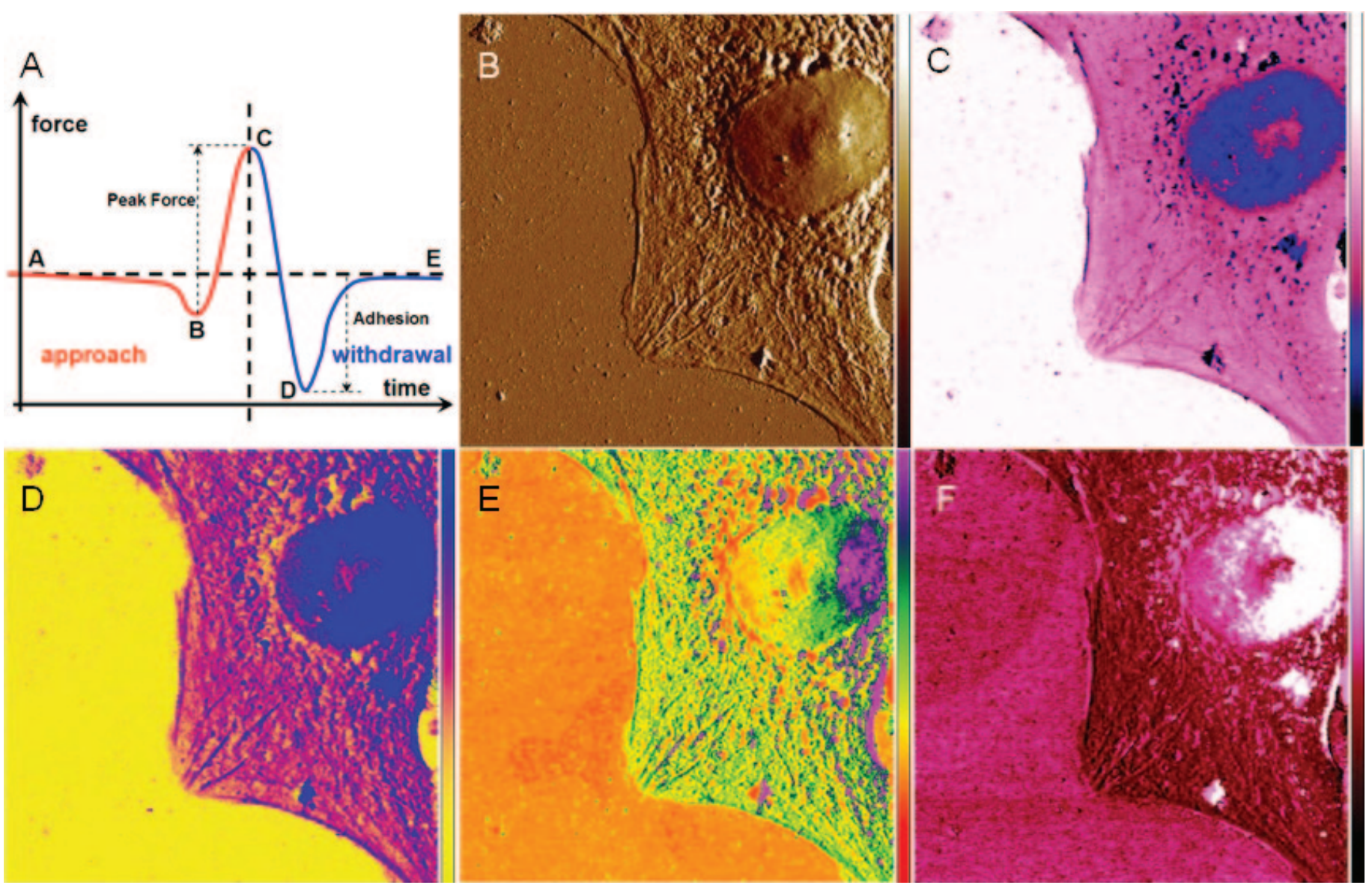

Figure 5: Peak Force QNM on fixed U2-OS cells. (A) "Heartbeat" profile of the tip going through contact and retraction over the time and showing the "peak force". At each pixel of the image, a force/distance curve (not shown here) is generated from which the different channels can be extracted. Adhesion is calculated like in regular force mode and is the maximum step height between the most negative point of the retract curve and the base line. On the contrary, peak force is the maximum step height between the most positive point of the extend curve and the base line. Dissipation is calculated by integrating the area between the two curves. Young's modulus is calculated by fitting the contact region of the retract curve using the DMT model. Deformation of the sample is obtained by comparison of the $z$-piezo motion and the cantilever deflection. The tip was calibrated on a known sample prior to experiment. Hence the $z$-scales are respectively $0-350 \mathrm{mV}$ for peak force error (B), 0-1000 kPa for DMT modulus (C), 0-5 nm for deformation (D), 0-850 eV for dissipation (E) and 0-250 nm for adhesion (F). Scan size is $55 \times 55 \mu \mathrm{m}$. 
scanned to extract the topography, stiffness, and proteinprotein unbinding information. Unbinding events were detected on the retraction curve, using an algorithm that finds events that are not related to noise [47].

\section{Results}

Figures 5 and 6 give representative examples of the potential of Peak Force QNM on fixed and living cells, respectively. Figure $5 \mathrm{~A}$ shows the force on the tip as a function of time for fixed cells. Because force/distance curves are generated at each pixel, images of peak force error, modulus, deformation, dissipation, and adhesion can be produced (Figures 5B through 5F). Figure 6 shows images of these same parameters for live cells. All cells were imaged using a probe calibrated on a sample having known mechanical properties, thus all the displayed channels show quantitative data. On fixed cells, good contrasts can be observed on most of the displayed channels, which reveals some interesting features of the subcellular network. The adhesion contrast between the cells and the support is not strong, which is in agreement with the fact that working in liquids greatly reduces the adhesion forces. The maximum dissipation occurs at the locations where the tip-sample contact is maximized (edge of the cell), though the differences in dissipation between the cell and the stiff support are clearly marked. This dissipation can be a mix of several factors like topography, elasticity, adhesion, and viscosity, but on the cell edges, topography is likely to be the major contribution to it.
Living cells exhibit the same tendencies, but the most remarkable differences between fixed and living cells are observed in topography, Young's modulus, and deformation. As shown in Figure 7, the topography of living cells is much higher $(1402 \pm 169 \mathrm{~nm})$ than fixed cells $(571 \pm 92 \mathrm{~nm})$. This can be explained by the fact that glutaraldehyde acts as a powerful cross-linking agent on surface proteins, which tends to stretch the cells and cause them to spread and flatten on the support. Second, living cells are considerably softer $(73.6 \pm 16.7 \mathrm{kPa})$ than fixed cells $(212.2 \pm 16.3 \mathrm{kPa})$. Third, living cells are much more deformable $(4.3 \pm 0.3 \mathrm{~nm})$ than fixed cells $(2.8 \pm 0.2 \mathrm{~nm})$.

\section{Discussion}

Topography and peak force error images (Figures 5B, 6A, and $6 \mathrm{~B}$ ) show image resolution similar to that obtained in tapping mode; the other channels display interesting contrast in the cell substructure. The DMT [48] modulus channel shows that the cells are several orders of magnitude softer than their substrate, and the difference of stiffness between the edges and the center parts of the cells is also highly contrasted (Figures 5C and 6C). The average Young's modulus is in agreement with previous studies on other types of cells [33]. Cells also appear to be easily deformable in their center but not so much on their edges (Figures 5D and 6D). The substrate should not play a significant role in such differences because even on their thinnest parts, cells are at least $50 \mathrm{~nm}$ high and by controlling the peak force, the indentation depth should not exceed a tenth
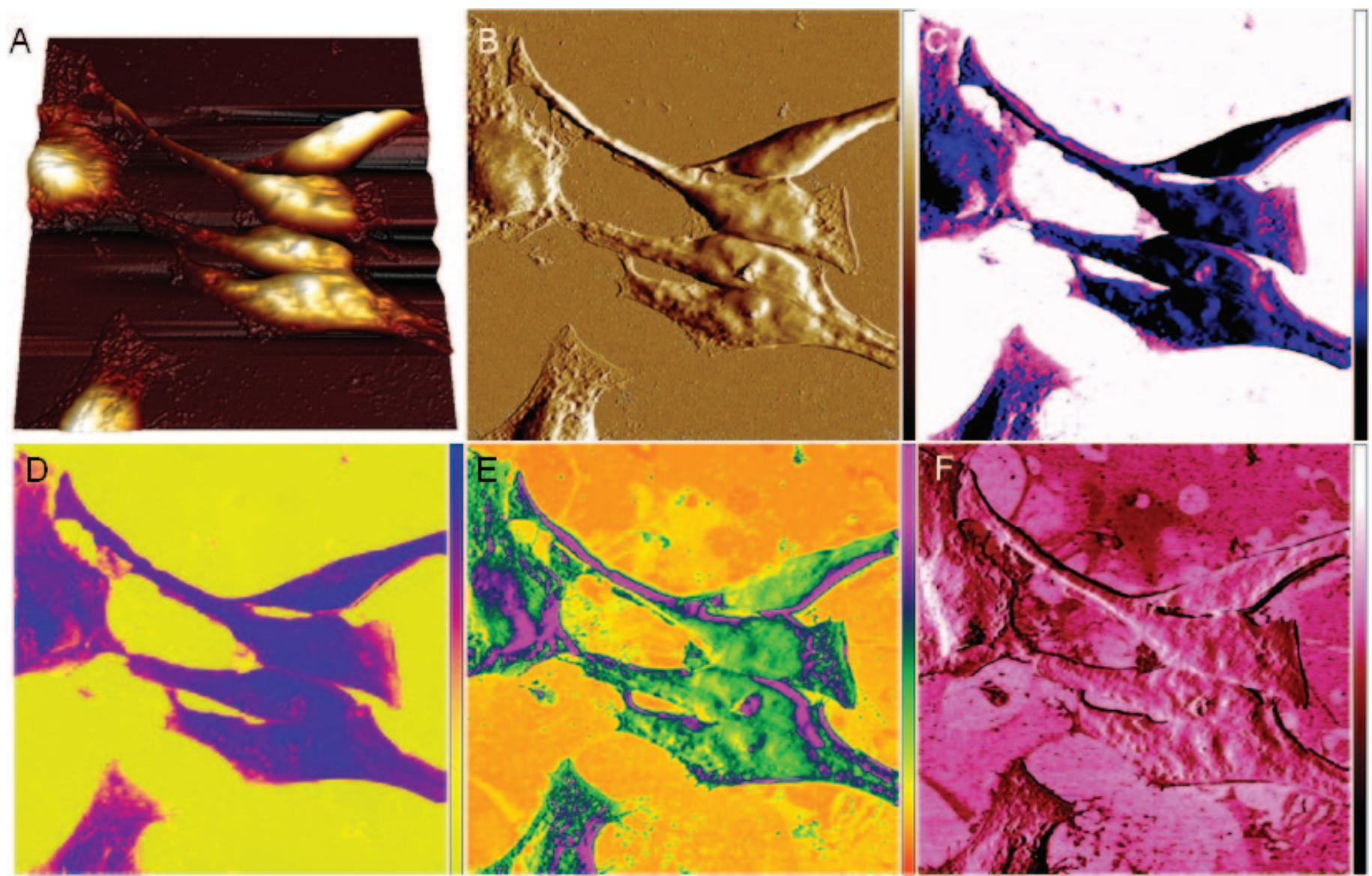

Figure 6: Peak Force QNM on living U2-OS cells. The probe was calibrated before the experiment. (A) shows a 3d-height representation of the scanned area. The $z$-scales are respectively 0-300 mV for peak force error (B), 0-700 kPa for DMT modulus (C), 0-5 nm for deformation (D), 0-850 eV for dissipation (E) and 0-250 $\mathrm{nm}$ for adhesion (F). Scan size is $100 \times 100 \mu \mathrm{m}$. 


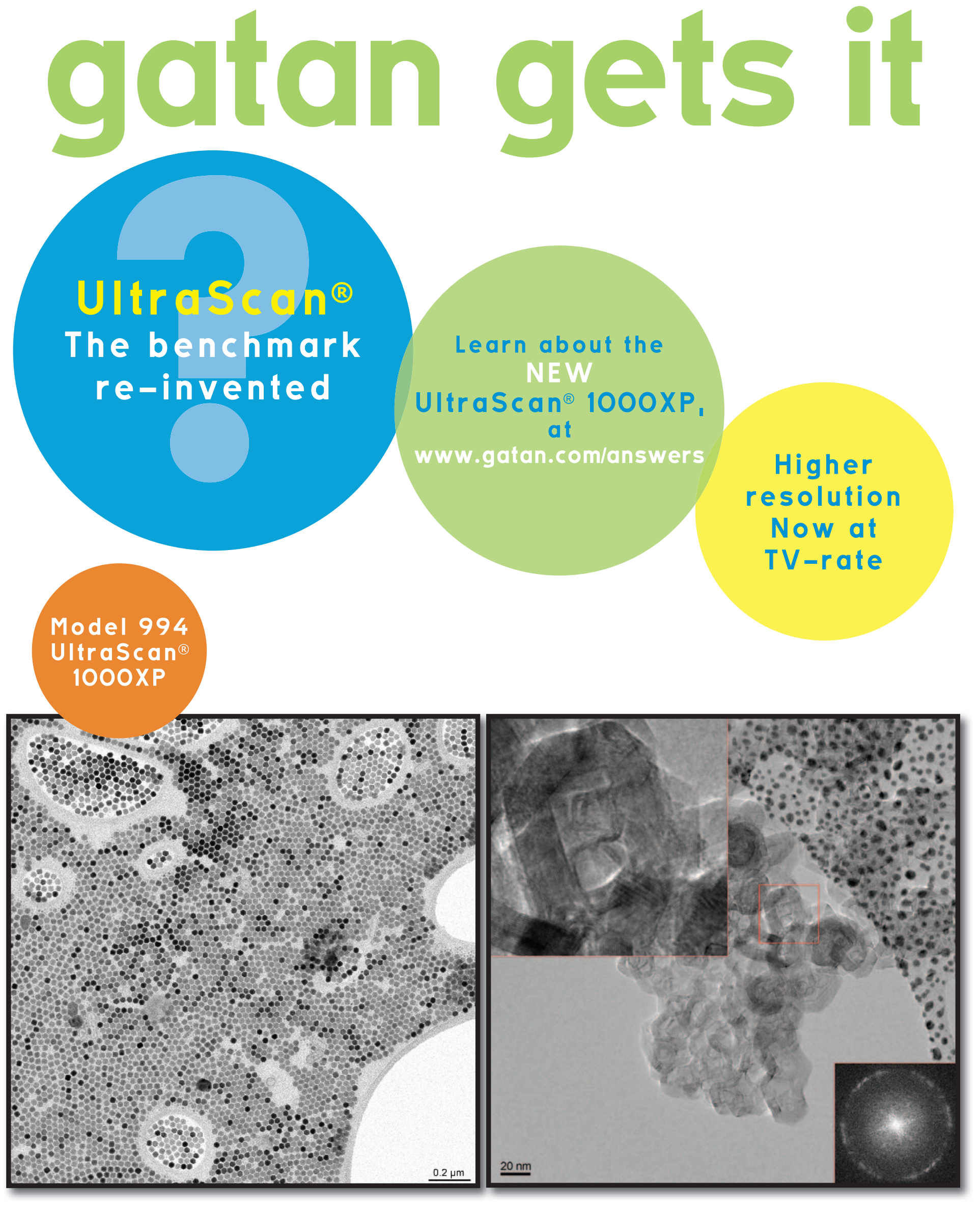

Left Image: Nano-crystals recorded with Gatan UltraScan ${ }^{\circledR}$ 1000XP CCD camera on a 120kV TEM. Image courtesy of Dr. Kent McDonald, University of California, Berkeley. Right Image: Graphitized carbon showing $0.34 \mathrm{~nm}$ lattice and FFT recorded with Gatan UltraS $\mathrm{San}{ }^{\circledR} 1000 \mathrm{XP}$ CCD camera (200kV),
Downloaded from https:/www.cambridge. org/core. University of Basel Library, on 30 May 2017 at 15:49:36, subject to the Cambridge Core terms of use, available at

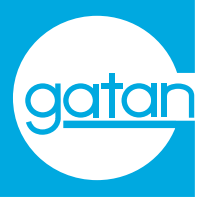



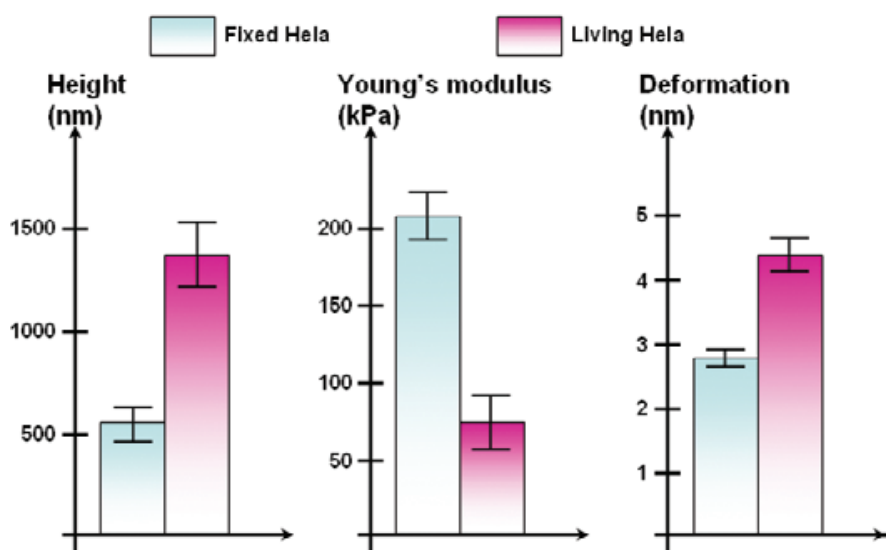

Figure 7: Statistical quantitative comparison of average height, Young's modulus, and deformation between fixed and living U2-OS cells. For each type of sample, three different areas were considered and calculation was done by using a bearing analysis on Peak Force Tapping images.

of nanometers. Dissipation-reflecting the energy dissipated between the tip and the sample during each tapping cycle-is strongly related to topography. Some local changes in contrast are visible on the cells, but the most striking contrasts are observed on the edges, which makes sense because at this place, the contact area between the probe and the sample is strongly increased (Figures 5E and 6E). Figure 7 shows that fixed and living cells exhibit dramatic differences in topography, elasticity, and deformability. As a comparison, similar experiments carried out in tapping mode would have probably revealed a high phase contrast between the cells and the support. However, such images would have been a contribution of several properties, whereas Peak Force Tapping mode offers the possibility of distinguishing between these different factors and quantifying them. This type of easy, quantitative approach offers new perspectives in many fields of biology, especially cancer research and physiology.

\section{Conclusion}

Since the first investigations on living cells in the early 1990s, AFM has improved considerably. Contact, tapping, and force modes now provide insight in fields like cancer research, infectious diseases, and tissue engineering. In addition, AFM may be a potential diagnosis tool for certain pathologies. The recent development of automated AFM and the emergence of new modes like Peak Force Tapping mode will likely make imaging of living cells and the acquisition of simultaneous quantitative information faster and easier.

\section{Acknowledgments}

We want to greatly thank Frank Lafont and Joelle Warrein (Pasteur Institute Lille, France) for providing the Hela cell samples and Liliane Glausser (Ecole Polytechnique Federale de Lausanne, Switzerland) for neuron preparation. C. Roduit is supported by the Indo-Swiss Joint Research Program (ISJRP), grand 122 941. Financial support by Ministerium für Wissenschaft, Forschung und Kunst - Baden-Württemberg (to $\mathrm{MH}$ ) is gratefully acknowledged.

\section{References}

[1] RA Lugmaier et al., Ultramicroscopy 104 (2005) 255-60.

[2] Z Deng et al., Biophys J 96 (2009) 1629-39.

[3] DJ Frankel et al., Biophys J 90 (2006) 2404-13.
[4] A Yersin et al., Biophys J 92 (2007) 4482-89.

[5] AE Brown et al., Biophys J 96 (2009) 1952-60.

[6] S Kasas and A Ikai, Biophys J 68 (1995) 1678-80.

[7] G Binnig et al., Phys Rev Lett 56 (1986) 930-33.

[8] Q Zhong et al., Surf Sci Lett 290 (1993) L688-L692.

[9] Z Shao et al., Adv Phys 45 (1996) 1-86.

[10] C Le Grimellec et al., Biophys J 75 (1998) 695-703.

[11] J Spatz et al., Nanotechnol 6 (1995) 40-44.

[12] HJ Butt et al., J Struct Biol 105 (1990) 54-61.

[13] J Hörber et al., Scanning Microsc 6 (1992) 919-29.

[14] C Le Grimellec et al., Biophys J 67 (1994) 36-41.

[15] E Evans and K Ritchie, Biophys $J 72$ (1997) 1541-55.

[16] A Berquand et al., Langmuir 21 (2005) 5517-23.

[17] A Berquand et al., Microscopy Today 18 (2010) 34-37.

[18] E Henderson et al., Science 257 (1992) 1944-46.

[19] JH Hoh and CA Schoenenberger, J Cell Sci 107 (Pt 5) (1994) 1105-14.

[20] NJ Tao et al., Biophys J 63 (1992) 1165-69.

[21] L Ponce et al., in Microscopy: Science, Technology, Applications and Education, eds. A Méndez-Vilas and J Díaz, Formatex, Badajos, Spain, 2010 (in press).

[22] V Dupres et al., Langmuir 25 (2009) 9653-55.

[23] ML Liu et al., J Exp Hematol 12 (2004) 793-97.

[24] DP Allison and MJ Doktycz, J Cell Mol Med 10 (2006) $847-56$.

[25] D Qiu et al., Biochem Biophys Res Commun 369 (2008) $735-40$

[26] J Lee et al., Nature 362 (1993) 167-71.

[27] TP Stossel, Science 260 (1993) 1086-94.

[28] C Rotsch et al., Proc Natl Acad Sci USA 96 (1999) 921-26.

[29] JA Dvorak and E Nagao, Exp Cell Res 242 (1998) 69-74.

[30] C Rotsch and M Radmacher, Biophys J 78 (2000) 520-35.

[31] A Schafer and M Radmacher, Acta Biomater 1 (2005) $273-80$.

[32] JC Martens and M Radmacher, Pflugers Arch 456 (2008) 95-100.

[33] X Cai et al., Scanning 31 (2009) 83-89.

[34] H Oberleithner et al., Proc Natl Acad Sci USA 104 (2007) 16281-86.

[35] Y Park et al., Proc Natl Acad Sci USA 105 (2008) 13730-35.

[36] I Sokolov, in Cancer Nanotechnology, eds. H Nalwa and T Webster, American Scientific Publishers, Valencia, CA, 2007.

[37] SE Cross et al., Nat Nanotechnol 2 (2007) 780-83.

[38] JP Mills et al., Proc Natl Acad Sci USA 104 (2007) 9213-17.

[39] S Suresh, Acta Biomater 3 (2007) 413-38.

[40] S Kumar and VM Weaver, Cancer Metastasis Rev 28 (2009) 113-27.

[41] H He et al., BMC Cell Biol 10 (2009) 81-92.

[42] HW Wu et al., Scanning 20 (1998) 389-97.

[43] I Sokolov et al., Nanomedicine 2 (2006) 31-36.

[44] I Dulinska et al., J Biochem Biophys Methods 66 (2006) $1-11$.

[45] C Roduit et al., Biophys J 94 (2008) 1521-32.

[46] FD Morgenthaler et al., Eur J Neurosci 17 (2003) 1365-74.

[47] C Roduit et al., Biophys J 97 (2009) 674-77.

[48] BV Derjaguin et al., J Colloid Interf Sci 53 (1975) 314-25. 
